\title{
Students' difficulties with partial differential equations in quantum mechanics
}

\author{
Tao Tu๑, ${ }^{*}$ Chuan-Feng Li, ${ }^{\dagger}$ Zong-Quan Zhou, and Guang-Can Guo \\ School of Physics, University of Science and Technology of China, Hefei 230026, People's Republic of China
}

(Received 30 March 2020; accepted 8 December 2020; published 21 December 2020)

\begin{abstract}
Upper-division physics students solve partial differential equations in various contexts in quantum mechanics courses. Separation of variables is a standard technique to solve these equations. We investigated students' solutions to midterm exam questions and utilized think-aloud interviews. We also applied a framework that organizes students' problem-solving process into four stages: activate, construct, execute, and reflect. Here we focused on students' problem-solving process for two typical problems in the context of quantum mechanics: an energy eigenfunction problem in two spatial dimensions and a time evolution problem in one spatial dimension. We found that the students encountered various difficulties when they used the separation of variables technique to solve these partial differential equations. Common difficulties included recognizing when separation of variables is the appropriate method, deriving the correct separated equations from the original equation, deciding the signs of the separation constants, justifying when using the summation form of the wave function, and using an effective reflecting tool for their final solutions. In addition, we observed qualitatively and quantitatively different errors in students' solutions to the two problems. Finally, we discussed the possible implications of our findings for instruction.
\end{abstract}

DOI: 10.1103/PhysRevPhysEducRes.16.020163

\section{INTRODUCTION}

There is a growing area of physics education research for the study of students' difficulties in quantum mechanics [1-27]. A substantial body of literature on students' difficulties has focused on the understanding of concepts and the formalism of quantum mechanics [10,11]. In quantum mechanics courses, students are often asked to use sophisticated mathematical techniques to tackle specific physics problems. In particular, how to solve partial differential equations (PDEs) appears frequently throughout quantum mechanics curricula. As far as we know, few studies discussed the use of mathematical tools during quantum physics problem solving [28,29].

There are several investigations on students' difficulties with solving ordinary differential equations (ODEs) in undergraduate mathematics courses [30-32]. Recently, there has been work on students' difficulties with the separation of variables technique to solve the Laplace equation in the context of upper-division electrostatics [33]. However, we are not aware of any existing research specifically targeting students' difficulties with differential

\footnotetext{
*tutao@ustc.edu.cn

cfli@ustc.edu.cn
}

Published by the American Physical Society under the terms of the Creative Commons Attribution 4.0 International license. Further distribution of this work must maintain attribution to the author(s) and the published article's title, journal citation, and DOI. equations in quantum mechanics. While a mathematical technique such as the PDE tool is general and reasonably context independent, the specific details of how a mathematical tool is used in physics problem solving is often highly dependent on the specific context [33-36]. Previous studies [36,37] also suggested that students' difficulties can perpetuate and new difficulties can occur when students transfer certain techniques of problem-solving from one context to another. For these reasons, more studies are needed to probe students' using a mathematical tool in different physics contexts, which would be potentially valuable to provide more insights $[33,34,36]$. The goal of this study is to identify how students' difficulties with mathematical tools of PDEs interact with their understanding of quantum physics content.

Students in the School of Physics at the University of Science and Technology of China (USTC) encounter PDEs several times in their quantum mechanics course, for example, in the context of solving Schrödinger equation for hydrogen atom in spherical coordinates. In discussions with the physics students at USTC, they demonstrated varying degrees of mastery of problem-solving techniques for PDEs in quantum mechanics course.

In the present work, we detect students' problem-solving skills when applying PDE tools in the context of quantum mechanics. Here, we specifically focus on two important cases: a time-independent Schrödinger equation (i.e., a PDE in two-dimensional space coordinates), and a timedependent Schrödinger equation (i.e., a PDE in onedimensional space coordinate and time coordinate). The 
two types of problems typically ask for the energy eigenfunctions of a particle in a region or an expression for the time evolution of the wave function. The two equations are solved with appropriate boundary conditions and initial conditions. The students were often asked to manipulate these equations into multiple ODEs using a mathematical technique known as separation of variables. Nevertheless, we do not claim that the study presented here covers all possible difficulties with differential equations in quantum mechanics but rather provides a sampling of students' difficulties when they encounter PDEs in quantum physics.

In this paper, we analyze investigation data through the lens of the activation, construction, execution, reflection (ACER) framework. This analytical framework was initially developed in the context of students performing computations for physics problems [34]. In Sec. II, we provide an overview of the related literature on students' difficulties with mathematics in the upper-division physics courses. In Sec. III, we describe the ACER framework to structure our investigations and analysis of students' difficulties. Section IV describes the context and methodology of the study. Then, in Secs. V and VI, we present our findings on students' difficulties in the use of mathematics tools for two different PDEs in quantum mechanics. Finally, in Sec. VII, we discuss the similarities and differences between our findings with previous studies of PDEs in other contexts [33] in detail. We also provide a brief discussion of implications for instruction and future work along the lines of the present paper.

\section{REVIEW OF LITERATURE}

In this section we present an overview of the previous literature related to our work.

\section{A. Mathematics in upper-division physics courses}

Physics education research initially focused on lowerdivision courses, and most efforts have probed students' thinking and reasoning of specific content from particular courses. Many studies typically have sought to identify students' difficulties in their understanding. In the last years, a subfield on upper-division courses has attracted the attention of an increasing number of physics education researchers [38].

As the content in upper-divisor physics courses becomes deeper, so does the demand for students' ability of using sophisticated mathematics tools. Therefore, researchers in physics education expanded the scope of their studies to explore how students use mathematics tools to learn the physics content and to solve the physics problems. There are some studies in specific physics content areas including classical mechanics [39], electrodynamics [4042], thermodynamics [43], and statistical mechanics [4446]. These studies have identified how students' difficulties with a general mathematics tool interact with their understanding of specific physics content.
Furthermore, a few studies have begun to find larger patterns across the various students' difficulties when they employ mathematical tools in upper-division physics courses. Bing and Redish investigated students' use of calculus in the context of classical mechanics [47]. They presented a system for classifying students' warrants and identified students' epistemological framings for using math in physics. Bajracharya and colleagues presented an analysis of students' use of partial derivatives in the context of thermodynamics [48]. They classified students' problem-solving strategies into two principal categories: the analytical derivation strategy and the graphical analysis strategy. In particular, Wilcox and colleagues developed an analytical framework to characterize students' difficulties when solving the long and complex problems in upperdivision physics courses [34,49]. They have applied this framework to analyze students' difficulties with specific mathematical techniques, such as integration [34], delta function [35], PDE [33], and boundary conditions [36] in the context of electricity and magnetism.

\section{B. Student learning in quantum mechanics content area}

Some of the physics education research in upper-division courses focused on students' understanding of quantum mechanics. Research in this area thus far has focused on probing the ability of students to learn and explain most of the basic quantum concepts: quantum interference phenomena [17-19], quantum tunneling [4], the properties of wave functions $[1,3,6,22,24]$, the time development and expectation values of a wave function $[1,3,6,13,21,26]$, measurement outcomes of various physical observables $[7,8,12]$, distinguishing between Euclidian space and Hilbert space [1,3], different notations (e.g., Dirac notation, algebraic wave-function notation, and matrix notation) [14,22,25], angular momentums [5,9], and uncertainty principle $[1,3,6]$. Research has found that students had various difficulties in mastering concepts and applying the formalism to answer qualitative questions related to these basic concepts.

Singh and Marshman synthesized and discussed students' reasoning difficulties in quantum mechanics that have been documented in previous studies [10]. They found that students' difficulties were common in distinguishing between closely related concepts in quantum mechanics. They suggested that students' difficulties were often due to overgeneralizing concepts learned in one context to another context where these concepts are not directly applicable. In addition, Marshman and Singh constructed a framework to characterize the patterns of students' conceptual and reasoning abilities in quantum mechanics [11]. The framework incorporates students' prior preparation, goals, motivation, and paradigm. They found that the patterns of reasoning difficulties in quantum mechanics are a striking resemblance to those found in introductory classical mechanics course. 


\section{PDEs in physics courses and research questions}

The studies discussed so far focused on students' difficulties with specific topics when answering nonalgorithmic problems in quantum physics. However, the research area on students' skills of problem solving in quantum mechanics is relatively unexplored. Learning quantum mechanics is challenging not only because the concepts of quantum mechanics and classical mechanics are very different but also because students struggle with increased mathematically sophisticated tools. An important context of quantum mechanics is solving algorithmic problems such as the Schrödinger equation (i.e., a typical PDE) with a complicated potential energy and boundary conditions. We are not aware of any studies that specifically focus on the student problem-solving process of PDEs in quantum mechanics.

There are some studies to investigate students' difficulties on solving ODEs in mathematics courses [30-32]. Recently, Wilcox and colleagues studied students' difficulties with PDE techniques to solve the Laplace equation in Cartesian and spherical geometries in the context of electrostatics [33]. They examined students' solutions following the ACER framework that divide students' problem-solving process into four stages: activation, construction, execution, and reflection. They found that students encountered various challenges in different stages. In the activation stage, students had difficulties recognizing when separation of variables is the appropriate tool. In the construction stage, students often failed to justify the separated form of the potential and the need for the infinite sum, or to identify implicit boundary conditions. In the reflection stage, students rarely spontaneously reflected on their solutions.

Despite this study [33], there remains a need for more investigations on students using PDE techniques in different physics contexts. On the one hand, previous studies suggested that the way in which a mathematical tool is used to solve a physics problem is often highly dependent on the specific context in which that tool is being used [33-36]. In order to solve a problem successfully, students are required to go back and forth between mathematics and physics: convert a physics situation to a mathematical expression, perform a long and complex mathematical procedure, and understand the physics significance of the result of mathematical calculation. In particular, in the viewpoint of the ACER framework, the application of PDE tools in a physics problem can be divided into four stages:

(i) Activation stage: recognize the PDE related tools.

(ii) Construction stage: set up the corresponding equations and conditions for the physics problem (e.g., decide the sign and the physics meaning of the separation constants to match the boundary conditions).

(iii) Execution stage: calculate the solutions to the equations and conditions. (iv) Reflection stage: check the final answer.

The use of PDE tools depends on the specific context of a physics problem in all four stages of the process, especially in the construction stage. Although the work by Wilcox and colleagues have investigated students' performance in the four stages [33], the results were limited to students' difficulties in the context of electrostatics.

On the other hand, as a mathematical tool appear several times in different physics contexts across upper-division courses, it becomes increasingly challenging when students use the tool in a more advanced situation. Previous studies also suggested that students' difficulties can persist from one context to another, or new difficulties can appear as students encounter new physical contexts [36].

Zwolak and Manogue investigated students' reasoning in upper-division electricity and magnetism in the context of the paradigms curriculum [37], where students were first exposed to the application of separation of variables in quantum physics before they took electricity and magnetism. Students had much experience with separation of variables in the context of quantum mechanics, before they saw it as part of electricity and magnetism. However, the researcher found that students did not choose the separation of variables technique as a natural problem-solving tool for PDEs when they departed from the quantum context. They also suggested that new difficulties can occur when students transfer certain mathematical techniques of solving problems from one physics context to another.

Because of these reasons, additional work is valuable to probe students' difficulties when utilizing PDE tools in other contexts, such as quantum mechanics. Our current study adds a new and interesting piece to the picture. Given the previous work that has been done [33] and our focus on student problem-solving of quantum mechanics, we are interested in three research questions:

(1) To what extent can students use PDE tools in the context of quantum physics? That is, when solving a Schrödinger equation in specific situations, how well do students correctly connect the elements of the solving procedure with the physics situations?

(2) What are the common difficulties that students encounter when solving a PDE in quantum mechanics? Can we categorize these difficulties? Can we find evidence of possible causes that lead to these difficulties?

(3) Compared to previous studies in other contexts, what difficulties are correlated and what difficulties are different?

\section{THEORETICAL FRAMEWORK}

Analysis of students' problem-solving procedure at the upper-division level is often a challenge. The procedure is often long and complex; thus, the students can encounter various difficulties and make errors at different stages of the process. To manage this complexity, we apply the analytic 


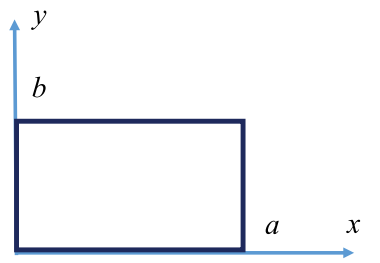

FIG. 1. An example of the exam problem about the energy eigenfunction.

framework known as the ACER framework to structure our investigations of students' works [34]. Using the ACER framework allows us to not only find common ideas on how students perform calculations but also understand in depth how the students interact with difficulties in the solution process.

According to the ACER framework, the problem-solving procedure is divided into four general stages: activation of mathematical tools related to the physics problems, construction of mathematical equations or models for the physics problems, execution of calculations step by step, and reflection on the final answers. These key elements were initially developed in the context of studying experts' problem solving [34]; then, these structural features were found to also impact students' performance of computations of various physics problems $[33,35,36]$. In the present work, we apply the general ACER framework to build a specific detailed outline of a correct solution to a particular PDE problem in quantum mechanics. The readers can find additional information about the process of operationalizing the ACER framework in Ref. [34].

We followed the scheme in Ref. [34] to manipulate ACER for a specific case. The authors of this paper listed the procedure of solving the PDE problems step by step and then classified these steps into the general stages of the ACER framework. The authors discussed and refined the outline until they all agreed that the key elements of the problem-solving procedure were addressed according to the ACER framework. This expert-designed scheme can be used as a reference structure to analyze students' work.

We operationalize the ACER framework for two problems shown in Figs. 1 and 2. The resulting outline is detailed below and is used to guide our analysis of students' work. For more information, please see in the Appendix A and B.

Activation of the tools.-The first stage of the ACER framework is to identify appropriate mathematical methods to solve the corresponding quantum mechanics problems.

- A1: The question provides boundary conditions and initial conditions and asks for the wave function with direct separation of variables [e.g., $\Psi(x, y)$ for problem 1 and $\Psi(x, t)$ for problem 2]

- A2: The question uses language associated with separation of variables [e.g., a sum expression of the initial condition $\left.\Psi(x, 0)=c_{1} \phi_{1}+c_{2} \phi_{2}\right]$

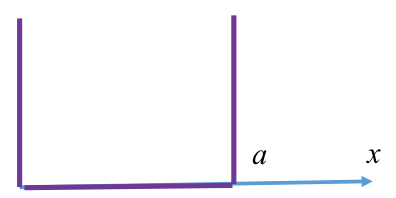

FIG. 2. An example of the exam problem about the time evolution of the wave function.

We find that two kinds of cues in a prompt are likely to activate the mathematical resources associated with these problems. For element A1, it is easier to use the associated separation of variables technique to manipulate the PDE. In addition, separation of variables gives a set of solutions, and it is typically to sum them to construct a general solution. For element A2, this specific aspect of a summation form can prompt students to use resources for the separation of variables method.

Construction of the equations.-Elements in this stage address the related equations and the general solutions so that they match the boundary conditions and initial conditions.

- C1: Express a relevant PDE for the problem (e.g., an energy eigenequation for problem 1 and a timedependent Schrödinger equation for problem 2).

- C2: Decide the sign of the separation constants or the function forms for the solutions that are appropriate for the boundary conditions and initial conditions.

- C3: Set up all the boundary conditions and initial conditions to the general solutions in order to determine all unknown constants.

Execution of the calculations.-This stage of the ACER framework executes the mathematical calculations to the equations and conditions built in the construction stage.

- E1: Use the separated form of the wave function to divide the PDE into ODEs [e.g., $\Psi(x, y)=f(x) g(y)$ for problem 1, $\Psi(x, t)=\phi(x) f(t)$ for problem 2].

- E2: Look up the solutions to these ODEs.

- E3: Apply the boundary conditions and initial conditions to determine the values for all unknown constants in the general solutions.

- E4: Organize the final answers to an interpretable expression for the wave function $\Psi$.

As noted in Ref. [33], step E3 can be accomplished using a variety of specific techniques, such as zero matching, term matching, and Fourier integrals, to address the boundary conditions and initial condition. These mathematical tools are explicitly discussed in Sec. V.

Reflection on the solutions. - The final stage of the ACER framework checks the final results.

- R1: Confirm whether the final answers satisfy the basic equation related to the problem.

- R2: Confirm whether the final answers match all boundary conditions and initial conditions.

- R3: Check whether the units of the final results are consistent. 
We note that R1 and R2 are particular reflection techniques for the present problems. Actually, we find that some students made mistakes in writing the final expressions that did not satisfy the basic equations and given conditions. We also note that the element R3 is a common technique for reflecting on a physical problem.

We will apply this ACER framework to study the students' work on PDE problems in quantum mechanics in the following sections.

\section{RESEARCH METHOD}

The research studies on students' difficulties summarized in this report use both quantitative and qualitative methodologies. We collected data from a one-semester quantum mechanics course at USTC. This course typically covers 12 chapters of Zeng's book [50] or 10 chapters of Griffiths' book [51]. The upper-division students in the school of physics took this course, with a typical class size of 60-110 students. In this study, we collected data from two distinct sources: students' solutions to questions in traditional midterm exams and "think-aloud" interviews for problem solving. We identified the common student difficulties from the quantitative data in their writing solutions for exams and gained deeper insight into the nature of those difficulties through their responses in the interviews.

\section{A. Written exams}

We all taught the quantum mechanics course during the data collection phase. We collected midterm exam data from the course over 6 years. We codesigned the questions on these exams through discussions. In each exam, the students were asked to solve a PDE question, and we analyzed 6 distinct exams. One question in our data provided the students with a given potential and asked them to find the energy eigenfunction results (e.g., Fig. 1). We collected $N_{t}=344$ solutions from 3 exams. The other question provided students with an initial wave function and asked them to find an expression for the time evolution of the wave function (e.g., Fig. 2). We collected $N_{t}=381$ solutions from another 3 exams.

\section{B. Design the interviews}

In our studies, we conducted "think-aloud" interviews [52] to investigate student's difficulties with problem solving in more depth and to unravel the possible underlying cognitive mechanisms. In these qualitative studies, a subset of students (the total number is smaller than that in the classroom written exams) were interviewed individually outside of the class. Students were told in advance that this was only an interview instead of a test and it would not be counted for one test grade. Thus, the students will not be nervous and they could better demonstrate their problemsolving skills. Almost all of the students disagreed with audio and video recording because they found it disturbing and felt a sense of being watched. Therefore, all interviews were transcribed verbatim. For consistency, all of the interviews were conducted by the first author of this paper.

The students interviewed were given the similar problems as those in the written exams because we wanted to probe their problem-solving strategies and to understand the underlying cognitive mechanism. During the interviews, we provided students with a pen and paper and asked them to verbalize their thought processes when they worked on the problems. These interviews were semistructured in the sense that we had a list of questions that we wanted to probe. These questions were not brought up initially in the interviews because we wanted students to formulate and articulate their thought processes by themselves. After the students had expressed their problemsolving strategies to the best of their ability, they were asked additional questions for clarifying certain issues more clearly.

Many questions were from the list of elements that we wanted to probe in accordance with the ACER framework. To probe the activation stage, we asked the students what prompted them to use PDE related resources. Then we asked the questions to understand how students modeled the relevant equations and performed the corresponding calculations (i.e., the construction and execution stage). Lastly, we examined how students who solved the problem in the allotted time checked their solutions (i.e., the reflection stage). From the perspective of the ACER framework, these interview questions clearly covered the entire process of the four stages. We asked students these questions during the second half of the interview if students did not mention these issues by themselves. Other questions were designed on the spot by the researcher to probe a particular student's thought process according to their response. In some interviews, we asked students more broad issues about what difficulties they faced in learning quantum mechanics course. They were not disturbed when they answered the questions, except we prompted them to keep talking if they were quiet for a long time.

Students' responses to interviews provided an additional data source. The first set of interviews were designed using the above semistructured, "think-aloud" protocol, and we collected $N=24$ solutions. The students were asked to determine the energy eigenfunctions for a particle inside a box (i.e., question 1 in Fig. 1). The second set of interviews were conducted in a similar way, and we collected $N=30$ solutions. In this case, the students were asked to find an expression of the wave function at any time (i.e., question 2 in Fig. 2).

\section{FINDINGS ON STUDENTS' DIFFICULTIES WITH A PDE IN AN ENERGY EIGENFUNCTION PROBLEM}

When we use a similar ACER framework to study the students' work on the two problems, we find that different 
kinds of errors occur in their work on the two problems. Thus, we report on students' difficulties with these two problems separately. In this section, we follow the stages and steps of the ACER framework to identify and analyze common difficulties in students' work with a PDE in an energy eigenfunction problem (question 1 in Fig. 1).

\section{A. Activation of the tools}

Energy eigenfunction problems are common in quantum mechanics courses, and they are usually solved by analytical methods using differential equations. Element A1 typically applies to high-dimensional energy eigenfunction problems. Since these problems provided boundary conditions such as the form $\Psi(x=a, y)=0$, they could prompt students to activate tools such as separation of variables related to PDEs. The majority of the exam solutions (97\%, $N=323$ of 344$)$ explicitly presented the appropriate separated form of the wave function [i.e., $\Psi(x, y)=f(x) g(y)]$. A very small fraction of the solutions (3\%, $N=11$ of 344) wrote an evolution operator (e.g., $\left.e^{-i \hat{H} t} \Psi\right)$ or a Hamiltonian operator (e.g., incorrectly wrote the expression $\hat{H} \Psi=E_{n} \varphi_{n}$ ) and attempted to make some transformations while not mentioning the separation of variables $x$ and $y$.

In the interviews, we directly asked students to comment on when separation of variables is a potential tool to obtain the wave function. The correct response is that this method is applicable in situations where the solution is specified on the boundaries of some region and the problem asks to find the solution inside the region. However, the students either made no response or could not articulate this subtle argument. One student answered, "We have learned various PDEs of three-dimensional space in the quantum mechanics course, such as for a hydrogen atom. Usually, we adopt the separation of variables for solving these PDEs." This result may indicate that our students often relate questions to previous similar problems when selecting separation of variables as preferred tool.

Another student wrote a general Hamiltonian operator notation and tried to do some transformations (e.g., $\left.\hat{H} \Psi=\hat{H} \sum_{n} c_{n} \varphi_{n}=\sum_{n} c_{n} E_{n} \varphi_{n}\right)$. Though he wrote the correct formula, however, he did not mention what were the explicit expressions of the energy eigenfunctions $\varphi_{n}$. When he was asked why he used this method, he explained, "In quantum mechanics courses, we always expressed an operator $\hat{F}$ to describe a physical quantity. The eigenvalues and eigenstates are obtained by acting the operator $\hat{F}$ on the system such as $\hat{F} \phi=\lambda \phi$." Students learned two types of formalism in quantum mechanics course: algebraic expressions in position representation, e.g., the Hamiltonian eigenequation $\left[-\left(\hbar^{2} / 2 m\right) \nabla^{2}+U(\vec{r})\right] \varphi(\vec{r})=E \varphi(\vec{r})$, and abstract expressions, e.g., the Hamiltonian eigenequation: $\hat{H} \varphi_{n}=E_{n} \varphi_{n}$ or $\hat{H}|n\rangle=E_{n}|n\rangle$. This result may suggest that some students overfocused on the operator method
TABLE I. Difficulties in step C1: setting up a basic equation for the energy eigenfunction problem. Note that there may be multiple errors in one student's answer, thus the sum of $N$ in the table need not be equal to the number of student solutions. This case is applicable to the following tables.

\begin{tabular}{lc}
\hline Difficulty & $N$ \\
\hline Attempting to use the time-dependent & 10 \\
$\begin{array}{l}\text { Schrödinger equation but failing } \\
\text { Other errors }\end{array}$ & 10 \\
\hline \hline
\end{tabular}

related to abstract formalism, which may discourage them to activate separation of variables method to solve a PDE in position representation.

\section{B. Construction of the equations}

Step C1: The construction stage maps the physical content of a problem to a series of equations. Step C1 is to model a basic equation for a problem that the solution can satisfy. For the present energy eigenfunction question, this step amounts to writing the eigenvalue equation for the Hamiltonian, i.e.,

$$
-\frac{\hbar^{2}}{2 m}\left(\frac{\partial^{2}}{\partial x^{2}}+\frac{\partial^{2}}{\partial y^{2}}\right) \Psi(x, y)=E \Psi(x, y) .
$$

$N=323$ solutions used separation of variables method, and $N=303$ solutions provided the correct expression, while the common errors in the remaining $N=20$ solutions included (see Table I): attempting to use a time-dependent Schrödinger equation but becoming lost along the way [e.g., writing down $i \hbar \partial / \partial t \Psi=-\left(\hbar^{2} / 2 m\right) \nabla^{2} \Psi$ but not reducing it to $-\left(\hbar^{2} / 2 m\right) \nabla^{2} \Psi=E \Psi$ ], or other errors (setting up the equation with incorrect constant factors, e.g., writing down $-i \hbar / 2 m$ instead of $-\hbar^{2} / 2 m$; adding inappropriate terms, e.g., $\partial^{2} / \partial^{2} z$; using an incorrect Laplace operator).

The most common difficulty with element $\mathrm{C} 1$ for the energy eigenfunction problem is a focus on the time-dependent Schrödinger equation. For example, in the interviews, one student wrote a time-dependent Schrödinger equation directly. He claimed, "The timedependent Schrödinger equation is considered the most fundamental equation of quantum mechanics." While this is true, he could not further apply separation of variables to obtain the time-independent Schrödinger equation, which is the eigenvalue equation for the Hamiltonian. Thus, overemphasis on the time-dependent Schrödinger equation in quantum mechanics courses may result in some students struggling with how to reduce it to the time-independent Schrödinger equation.

Step C2: In practice, the students explicitly worked through the process of separation of variables (step E1) and then had to choose the separation constants for the ODEs that can satisfy the boundaries, i.e., 
TABLE II. Difficulties in step C2: determining the separation constants.

\begin{tabular}{ll}
\hline \hline Difficulty & $N$ \\
\hline Selecting two separation constants with negative values & 17 \\
Selecting one with a positive value and one with & 14 \\
$\quad$ a negative value) & 4 \\
Or not recognizing the need to determine the & \\
$\quad$ sign of the separation constants & \\
\hline \hline
\end{tabular}

$$
-\frac{\hbar^{2}}{2 m} \frac{1}{f} \frac{\partial^{2}}{\partial x^{2}} f=E_{1}, \quad-\frac{\hbar^{2}}{2 m} \frac{1}{g} \frac{\partial^{2}}{\partial y^{2}} g=E_{2},
$$

where $E_{1}$ and $E_{2}$ are the separation constants. Using this strategy, step $\mathrm{C} 2$ requires deciding the positive sign of the separation constants, and thus, the solution of the ODEs obtains sinusoidal dependence. $N=279$ solutions provided the exact ODEs, and $N=244$ solutions indicated that both separation constants are positive such that they are consistent with the boundary conditions. The common errors in the remaining $N=35$ solutions included (see Table II): selecting two separation constants with negative values; selecting one with a positive value and one with a negative value, or not recognizing the need to determine the sign of the separation constants.

The interviews provided additional insight into students' difficulties in identifying the signs of the two separation constants. One of the students stated, "Both the separation constants $E_{1}$ and $E_{2}$ need negative values." When he was asked why he decided the negative value, he argued, "This is a bound state problem and the energy should be lower than zero." He also explained, "When a particle is in a state such that the energy is less than the potential energy at both plus and minus infinity, the particle is in a bound state." He focused on satisfying the bound state picture while failing to consider that the potential energy at both plus and minus infinity is also infinity in the present problem. This result suggests that students who argued for negative values on the exams may overgeneralize the context of bound states.

Another student selected the two separation constants with different signs. When he was asked why he made this choice, he answered, "When we solve many PDEs such as the Laplace equation, we always find that one separation constant is a positive value and the other is a negative value. This is a general case." This result may reflect that many students solved problems by recalling a similar problem but were not able to make corresponding modifications to the new situations.

Step C3: In the final element of the construction stage, one can apply boundary conditions to create equations to determine unknown constants in a general solution. $N=$ 226 solutions used the general solution of the PDE, and $N=199$ solutions set up accurate equations to match the boundary conditions. The common errors in the remaining
TABLE III. Difficulties in step C3: setting up expressions to match the boundary conditions.

\begin{tabular}{lc}
\hline \hline Difficulty & $N$ \\
\hline Inappropriately setting up a superposition expression & 18 \\
Not setting up an integral for the normalization constant & 7 \\
Other errors & 7 \\
\hline \hline
\end{tabular}

$N=27$ solutions included (see Table III): inappropriately setting up a superposition expression [e.g., using $\sum_{n} c_{n} f(x=a) g(y)=0$, where $f(x)$ and $g(y)$ are the solutions to the separated ODEs], not building an integral for the normalization constant, or other errors [putting boundary conditions on the wrong side, e.g., $f(y=0)=0$ instead of $f(x=0)=0$; missing a term in the expression].

The most common difficulty identified in the interviews was consistent with the students' performance on exams. Two students included a sum in their expression to match the boundary conditions [e.g., $\sum_{n} c_{n} \sin \left(k_{1} a\right) \sin \left(k_{2} y\right)=0$, or $\left.\sum_{n} c_{n} \sin \left(k_{1} x\right) \sin \left(k_{2} b\right)=0\right]$ and found that the coefficients $c_{n}$ would be arbitrary. Then, they were confused about this result and did not know how this expression would help them to go further. One of the participants stated, "The general solution should be a superposition of the energy eigenfunctions. I recalled that I used a sum of the eigenstates in my previous homework." However, he could not recognize that it is not necessary to introduce the sum for the present problem. One possible explanation for this issue is that many students only remembered an algorithm of previous problems but did not understand the reason for introducing a sum expression in the previous problems.

Another interview student did not set up an integral expression for the normalization constants. Then, he found that there are two unknown constants in the general solution. He argued, "Some constants can be arbitrary in the final solution." Thus, recognizing the boundary condition can be used not only for solving the unknown constants (e.g., eigenvalues of this type of problem), but also for normalization, which was a stumbling block for some students.

\section{Execution of the calculations}

Step E1: In the execution stage, one should work through the mathematical procedure of the equations set up in the construction stage. Step E1 substitutes the separated form of the wave function [i.e., $\Psi(x, y)=f(x) g(y)$ ] to divide the PDE into two ODEs in the $x$ and $y$ directions. $N=303$ solutions started from the energy eigenvalue equation and attempted to derive the ODEs. $N=279$ solutions successfully completed this process. The common errors in the remaining $N=24$ solutions included (see Table IV) incorrectly separating the PDE into ODEs (obtaining the separated expression but not recognizing that it implies that the 
TABLE IV. Difficulties in step E1: executing the procedure of dividing the PDE to ODEs.

\begin{tabular}{lc}
\hline \hline Difficulty & $N$ \\
\hline Incorrectly separating the PDE into ODES & 15 \\
Other errors & 9 \\
\hline \hline
\end{tabular}

two terms must be both constants, for instance, not applying the form

$$
-\frac{\hbar^{2}}{2 m} \frac{1}{f} \frac{d^{2} f}{d x^{2}}-\frac{\hbar^{2}}{2 m} \frac{1}{g} \frac{d^{2} g}{d y^{2}}=E
$$

to obtain $E_{1}+E_{2}=E$, where

$$
\text { and } \quad E_{2}=-\frac{\hbar^{2}}{2 m} \frac{1}{g} \frac{d^{2} g}{d y^{2}}
$$

are the separation constants; incorrectly using separation constants before deriving the fully separated expression, e.g., incorrectly arguing

$$
\begin{aligned}
& -\frac{\hbar^{2}}{2 m} \frac{d^{2} f}{d x^{2}} g=E_{1}, \\
& -\frac{\hbar^{2}}{2 m} f \frac{d^{2} g}{d y^{2}}=E_{2}
\end{aligned}
$$

or other errors, e.g., writing down the separated form of the wave function but not knowing how to continue; missing a term in the expression.

In the interviews, nineteen participants correctly used the separation form assumption and worked through the process of separating the PDE into the ODEs. However, when they were asked the purpose of assuming the separated form, more than half of the participants directly stated that they recalled a similar procedure in the quantum mechanics courses. For example, one student emphasized, "The separated functional form is used to deal with the hydrogen atom problem." Another student also said: "I recall that there was an explicit procedure for solving these problems. Now I was able to reproduce it." Then we asked the students more broad issues about how they remembered or mastered the various methods in learning quantum mechanics course. From their responses, we found the following fact: to prepare the exams, some students organized a list of important methods from their homework. Each of these methods corresponds to a specific problem. For example, the separated of variables method for a hydrogen atom problem; the creation and annihilation operator method for a harmonic oscillator problem; summation expression method for a time evolution problem. Then they tried to remember these problems and associated methods. On the exams, they would search their memory and use pattern matching to map the exam questions to similar problems in their memory. Therefore, the interviews may indicate that some students simply remembered the algorithm for manipulating the separation of variables rather than understood the motivation for making this separation form assumption. We note that in the study of the Laplace equation in the context of electrostatics, students also did not have a clear understanding of the mathematical sense of the separation of variables [33].

Step E2: This step attempts to solve the ODEs derived from separating the PDE (i.e., $d^{2} f / d x^{2}+k_{1}^{2} f=$ 0 and $d^{2} g / d y^{2}+k_{2}^{2} g=0$, where $k_{1}=\sqrt{2 m E_{1} / \hbar^{2}}$ and $\left.k_{2}=\sqrt{2 m E_{2} / \hbar^{2}}\right) . N=244$ solutions included the proper ODEs, and $N=226$ solutions provided the correct general solution of the ODEs. Various mistakes in remaining $N=$ 18 works contained the following: using a real exponential expression for the solution, e.g., $f(x)=e^{k_{1} x}$, attempting to solve the ODEs but getting lost along the way or obtaining an incorrect solution, using separation constants rather than their square roots in the solution, e.g., $f(x)=\sin \left(\frac{2 m E_{1}}{\hbar^{2}} x\right)$, providing the same expression in both the $x$ and $y$ directions, writing down a general solution but not plugging in the specific separation constant.

In practice, given that there was little information for the solution of the ODE, it became particularly important that students were able to perform the mathematical calculations for this solution (although the ODEs both had the solutions in the relatively simple form as sinusoidal function). In the interviews, fourteen participants directly wrote down the solutions and claimed that they remembered the solutions to these ODEs. The remaining students struggled to solve the ODEs but failed to obtain the correct solution. These linear second-order ODEs with constant coefficients were usually taught in mathematical courses, but the students rarely replicated the derivation in exams or interviews. Thus we assume that many students solved these ODEs by remembering the solutions rather than by executing the mathematical procedure.

Step E3: This step determines the values of the unknown constants in the general solution using the equations set up in step C3 through mathematical calculations. $N=199$ solutions contained the correct expression for unknown constants, and $N=185$ solutions obtained the correct results. In addition, there are various mathematical mistakes in the remaining $N=14$ solutions: losing or adding a constant factor, incorrectly writing $n^{2}$ as $n$, or not finishing the integral calculations for the normalization constant.

Step E4: After solving the constants for the general solution, step E4 compiles all context of the solution into a single expression, which is the final result for the wave function. $N=185$ solutions finished step $\mathrm{E} 3$, and $N=179$ solutions obtained the precise final expression. The remaining $N=6$ solutions included several mathematical errors: dropping or adding constant factors, using $n^{2}$ instead of $n$ in 
the solution, incorrectly simplifying the sinusoidal function, or not compiling a final expression.

\section{Reflection on the solutions}

The reflection stage checks the final expression. An expert usually checks whether the final answer satisfies the basic equation or matches the boundary conditions (i.e., elements R1 and R2). In particular, he or she can evaluate the result through the units on the left- and right-hand sides of an expression (i.e., element R3). Many of our students attempted to review their working. However, we cannot know the number of the students who conducted this reflection on their exams since many students did not write down this reflection process explicitly in their solutions.

On the one hand, in the interviews, all the participants who obtained the final results spontaneously reflected on their solutions. For example, one of them answered, "I have already reviewed the working two times, and now I am sure it is correct." As far as we know, many Chinese students usually review their working before finishing the exam. This habit was developed through various examinations over the years. On the other hand, many of our students in interviews claimed that they reflected on their solutions by checking the expressions step by step. Additionally, in the interviews, after he was directly prompted using element R2, one student answered, "I found that a check of the solution to match the boundary conditions is the most effective method. I did not realize this before!" After he was directly prompted using element R3, another student answered, "This was the first time that I attempted to check whether the unit of the expression is self-consistent. I never used this method to determine whether an expression is correct before." In summary, our students often reviewed their working spontaneously; however, the investigations suggest that they rarely used the effective reflective methods used by experts (e.g., elements R1, R2, and R3).

\section{E. Overview of students' performance}

$N_{t}=344$ students took the exams and were required to solve the eigenfunction problem. For clarity, in Fig. 3 we show the correctness of each step as students progressed through this problem. The histogram represents the number of correct solutions at each step. The dot labels the corresponding correct percentage, which is the fraction of the number of correct solutions $N$ with respect to the number of total solutions $N_{t}=344$. Ultimately, only $52 \%$ ( $N=179$ of 344) successfully worked through 8 steps of the problem and finished the correct final results. As students worked through the problem, the number of correct solutions decreases continuously, which means students encountered various difficulties in each step. The number of correct solutions decreases relatively more pronounced in step $\mathrm{C} 2$ and $\mathrm{C} 3$, which means there were more issues in these two steps. Thus, this result indicates

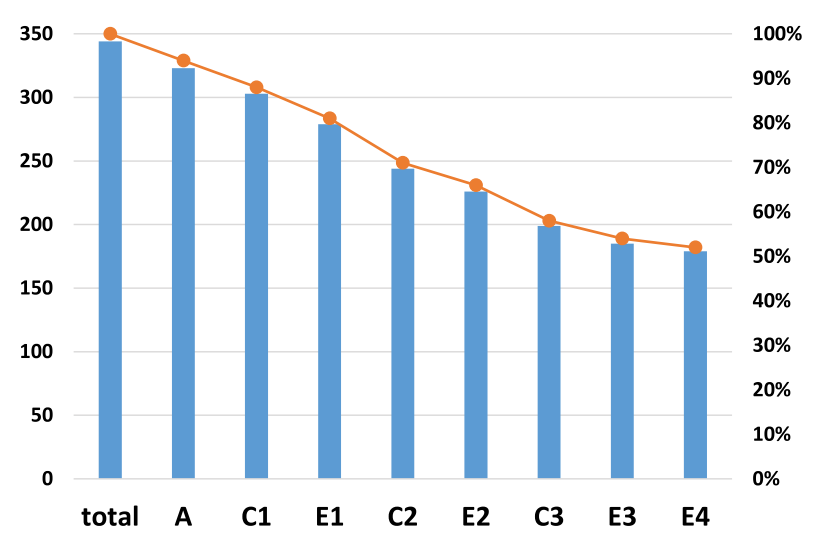

FIG. 3. The correctness of each step as students progressed through the energy eigenvalue problem. In the graph, the histogram denotes the number of correct solutions, and the dots label the correct percentage with respect to the total solutions.

that the construction stage involving the understanding of separation constants and superposition state expressions was a significant barrier to our students' success.

\section{FINDINGS ON STUDENTS' DIFFICULTIES WITH A PDE IN A TIME EVOLUTION PROBLEM}

In this section we provide our investigations of students' difficulties when dealing with a PDE in a time evolution problem for a particle in one-dimensional potential. The data and analysis are organized by the stages and elements according to the ACER framework utilized in Sec. III.

\section{A. Activation of the tools}

Most of the exam solutions (93\%, $N=355$ of 381) utilized the separation of variables method. The common alternative $(6 \%, N=21$ of 381$)$ was directly using evolution expression for a general wave function without work [e.g., $\left.\Psi(x, t)=\sum_{n} c_{n} e^{-i E_{n} t / \hbar} \varphi_{n}(x)\right]$. Nevertheless, the text in this question clearly instructs students to not directly write down the general expression for the wave function at time $t$ but instead provide the detailed derivation. Thus, these students who ignored these instructions had difficulties using the related tools for the time evolution problem. The remaining solutions $(1 \%, N=5$ of 381) wrote an evolution operator and attempted to made some transformations but failed.

The interviews provided additional insight into how the students activated related resources for the time evolution problem. One student provided a time-dependent Schrödinger equation and commented, "I only remember the general solution is $\Psi(x, t)=\sum_{n} c_{n} e^{-i E t} \varphi_{n}(x)$ but I can not recall how to obtain this expression." The student gave the wrong expression since $E$ should include the index $n$. This result reflects that in common quantum mechanics courses, a great deal of emphasis is placed on directly using a general expression for an evolution wave function and 
less is placed on using separation of variables to address a time-dependent Schrödinger equation. This approach may discourage students from understanding the origin of this expression and using separation of variables to solve the time-dependent Schrödinger equation.

The other student also provided a time-dependent Schrödinger equation but did not continue past this point. When he was asked to consider separation of variables, he answered, "I remember in the quantum mechanics course, the separation of variables method is used to solve PDEs in three spatial coordinates. I am hesitant to use this method for the PDE involving temporal coordinate." This result may suggest that some students did not recognize separation of variables as a general method to address PDEs. When they activated resources for problem solving, they often linked a method to a specific problem rather than to a class of general problems.

\section{B. Construction of the equations}

Step C1: The construction stage maps a physics problem to a mathematical model. Step C1 establishes the basic equation (i.e., a time-dependent Schrödinger equation), which is implicit in the prompt of the time evolution problem. $N=355$ solutions used separation of variables method, and $N=333$ solutions provided the correct expressions for a time-dependent Schrödinger equation, i.e.,

$$
i \hbar \frac{\partial}{\partial t} \Psi(x, t)=-\frac{\hbar^{2}}{2 m} \frac{\partial^{2}}{\partial x^{2}} \Psi(x, t) .
$$

The remaining $N=22$ solutions included various errors in this step (see Table $\mathrm{V}$ missing or adding a constant factor or sign (e.g., writing down $\hbar^{2} / 2 m$ instead of $-\hbar^{2} / 2 m$ ), directly writing down a time-independent Schrödinger equation, or other errors (omitting the $i$ factor; incorrectly expressing the time term, e.g., $\partial^{2} \Psi / \partial t^{2}$ instead of $\partial \Psi / \partial t$; expressing $\nabla^{2}$ in the $y$ and $z$ directions).

These findings are also consistent with the interview results. One participant wrote down a time-independent Schrödinger equation instead of a time-dependent Schrödinger equation. He explained, "We often used the time-independent Schrödinger equations to solve the eigenvalues and the time evolution of the wave function can be given directly by the final expression as

TABLE V. Difficulties in step C1: setting up a basic equation for the energy eigenfunction problem.

\begin{tabular}{lc}
\hline \hline Difficulty & $N$ \\
\hline Missing or adding a constant factor or sign & 10 \\
Directly writing down a time-independent & 8 \\
Schrödinger equation & 5 \\
Other errors & 5 \\
\hline
\end{tabular}

TABLE VI. Difficulties in step C2: determining the separation constants.

\begin{tabular}{lc}
\hline \hline Difficulty & $N$ \\
\hline Selecting the separation constant with a negative value & 25 \\
Not commenting on the sign of the separated constant & 2 \\
\hline \hline
\end{tabular}

$\Psi(x, t)=\sum_{n} c_{n} e^{-i E t} \varphi_{n}(x) . "$ We note that he also omitted an index $n$ for $E$ in this final formula. We hypothesize that this issue is related to difficulties in the activation stage, as some students used memory to guide equation building rather than understanding each step of the separation of variables method.

Step C2: This step determines the separated constant and identifies its physical meaning. $N=323$ solutions derived the exact ODEs from a time-independent Schrödinger equation [i.e., $i \hbar d f / d t=E f,-\left(\hbar^{2} / 2 m\right) d^{2} \varphi / d x^{2}=E \varphi$, where $E$ is the separation constant], and $N=296$ solutions explicitly commented that the separated constant is positive such that it is consistent with the boundary conditions. The common errors in the remaining $N=27$ solutions included (see Table VI): either selecting the separation constant with a negative value or not commenting on the sign of the separated constant.

In the interviews, twenty-two students expressed that the separation constant is energy $E$ and should be given a positive value. However, when they were asked why the separation constant is energy, only half of the students correctly answered that the ODE in the $x$ direction is an eigenvalue equation of the Hamiltonian; thus, the separated constant corresponds to the eigenvalue of energy. The other students could not explain it at all or simply argued that they used the symbol $E$ from memory. This result suggests that many students simply remembered every step of the algorithm and applied this logic to exams but did not truly understand the physical meaning of each step.

Step C3: This step combines a general solution with the boundary conditions and initial conditions to set up the equations to determine the unknown constants. $N=$ 288 solutions exploited the correct general solution, and $N=261$ solutions provided the correct equations to match the boundary conditions and initial condition. The common errors in the remaining $N=27$ solutions included (see Table VII): problems in expressing the superposition state to match the initial condition [e.g., using a single separated function $\sin (n \pi x / a)$ but not introducing a summation $\sum_{n} c_{n} \sin (n \pi x / a)$ ], incorrect belief that the time evolution of a wave function is always via an overall phase factor $e^{-i E t / \hbar}$, or other errors [not plugging in $x=a$ to match the boundary condition; not including the spatial function $\varphi(x)$ when matching the initial condition; not using the boundary conditions; not using the initial condition]. 
TABLE VII. Difficulties in step C3: setting up expressions to match the boundary conditions.

\begin{tabular}{ll}
\hline \hline Difficulty & $N$ \\
\hline $\begin{array}{l}\text { Problems in expressing the superposition state to } \\
\text { match the initial condition }\end{array}$ & 20 \\
$\begin{array}{l}\text { Incorrect belief that the time evolution of a wave } \\
\text { function is always via an overall phase factor } e^{-i E t / \hbar}\end{array}$ & 12 \\
Other errors & 11 \\
\hline \hline
\end{tabular}

In the interviews, twenty participants correctly wrote the general solution of the PDE [i.e., $\Psi(x, t)=$ $\left.\sum_{n} c_{n} e^{-i E_{n} t / \hbar} \sin (n \pi x / a)\right]$ and applied it to correctly set up the equation to match the initial condition, e.g.,

$$
\begin{aligned}
\sum_{n} c_{n} \sin \left(\frac{n \pi x}{a}\right) & =\Psi(x, t=0) \\
& =\sqrt{\frac{8}{5 a}}\left(1+\cos \frac{\pi x}{a}\right) \sin \frac{\pi x}{a} .
\end{aligned}
$$

Then, they were asked why they chose this superposition form of the wave function. The correct answer required the students to recognize that the Schrödinger equation is a linear differential equation and to transfer the physics content (i.e., the superposition principle of the wave function) to a corresponding mathematical expression (i.e., a superposition form of the general solution). However, their answers were unsatisfactory. For example, one interviewee commented, "We often used the superposition states for time development problems." This reply may indicate that many students approached these time evolution problems with a strategy that mapped the solution to the previous expression rather than deriving and justifying the key step.

One of the remaining students tried to utilize the initial conditions but failed. When he was asked how to use the initial conditions, he answered, "The general solution should be valid at every time. However, I found that the general solution does not match the initial conditions

$$
\sin \left(\frac{n \pi x}{a}\right) \neq \Psi(x, t=0)=\sqrt{\frac{8}{5 a}}\left(1+\cos \frac{\pi x}{a}\right) \sin \frac{\pi x}{a} .
$$

I guess there are some error in my previous expressions." He obtained the correct general solution in the previous step. Then, he was confused when he found that a single general solution is inconsistent with the initial conditions. However, he did not consider the possibility of combing a set of general solutions in a way that does satisfies the initial condition. Thus, this interview finding suggests that some students did not understand the critical step of the separation of variables method: a general solution for a PDE can be a superposition form in which each component can not satisfy the boundary conditions and initial conditions.
Another student jumped straight to the final expression with the formula $\Psi(x, t)=e^{-i E t / \hbar} \Psi(x, 0)$. When he was required to justify the expression, he said, "The time dependence of a stationary state is via a phase factor $e^{-i E t / \hbar}$. Since the wave function for this problem is a stationary state, it should have the expression $e^{-i E t / \hbar} \Psi(x, 0)$." This result suggests that some students focused on the form for a single stationary state, which may have prevented them from understanding the crucial step of the separation of variables method: using a summation of single solutions to construct a general solution.

\section{Execution of the calculations}

Step E1: The execution stage works through the mathematical procedure of the model built in the construction stage. Step E1 separates a time-dependent Schrödinger equation into ODEs by assuming a separated form of the wave function [i.e., $\Psi(x, t)=\varphi(x) f(t)$ ]. $N=333$ solutions began with a time-dependent Schrödinger equation, and $N=323$ solutions derived correct ODEs. The remaining $N=10$ solutions included beginning with a separated form of the wave function but failing to continue; making various mistakes in derivation and thus not obtaining the fully separated expression; not recognizing that the separated expression means a constant, i.e., the students did not relate the form

$$
i \hbar \frac{1}{f} \frac{d f}{d t}=-\frac{\hbar^{2}}{2 m} \frac{1}{\varphi} \frac{d^{2} \varphi}{d x^{2}}
$$

to introduce a separation constant $E$.

In the interviews, the participants wrote down the separation form of the wave function and attempted to manipulate the PDE to ODEs. Twenty-six students worked through this process, and the remaining student struggled in this process and ultimately failed. When one of these students was asked how to continue, he answered, "I recall that this content was taught at the beginning of the semester, but I cannot replicate it." Students typically learned this derivation only once in the quantum mechanics course, in the section on stationary state problems. Then, they often directly used a general expression of the time evolution wave function [i.e., $\Psi(x, t)=$ $\sum_{n} c_{n} e^{-i E_{n} t / \hbar} \varphi_{n}(x)$, where $\varphi_{n}$ is the energy eigenfunction] and rarely grasped the origin of this expression. Therefore, they may not have had the motivation to derive this expression in exams.

Step E2: This step yields a general solution for two ODEs. $N=296$ solutions used the correct ODEs, i.e.,

$$
i \hbar \frac{d f}{d t}=E f, \quad-\frac{\hbar^{2}}{2 m} \frac{d^{2} \varphi}{d x^{2}}=E \varphi,
$$

and $N=288$ solutions obtained the correct solutions to the ODEs. Various errors in the remaining $N=8$ solutions 
included omitting the $i$ factor in the temporal function, giving an incorrect expression for the spatial function, mixing the separation constant and its square root in the expression, inappropriately including an index $n$ in the expression, or trying to solve the ODEs but failing to complete this process successfully.

In the interviews, twenty-two participants correctly obtained the solutions for two ODEs in the spatial coordinate $x$ and the temporal coordinate $t$. One of the remaining students wrote down the expression $e^{-i E_{n} t / \hbar} \varphi(x)$. When he was asked why he used the label $E_{n}$ for the time part, he answered, "I remember that in the final answer the energy should have a subscript $n$." It is obvious that he confused the final answer and the solution in the problem-solving process. Actually, the solutions for the ODEs include only the separation constant $E$ without a subscript $n$, and this quantum number $n$ should be introduced in the next step C3. We also note that he provided the wrong final answer, as the quantum number $n$ should appear in the temporal part and spatial part of the correct final result simultaneously. Thus, we suspect that many students did not solve the ODEs, but simply remembered the solutions to these ODEs. If their memories were wrong, their answers were also wrong.

Another student provided a solution $e^{-i E t / \hbar}$ for the temporal equation; then, he quickly finished his solution by writing down the expression "the wave function at time $t$ is $\Psi(x, t)=e^{-i E t / \hbar} \Psi(x, 0)$." As noted in Ref. [10], students often struggled to distinguish the expression $e^{-i E t / \hbar} \varphi(x)$ for a single stationary state and the expression $e^{-i E_{n} t / \hbar} \varphi_{n}(x)$ for each component in a superposition state. The superficial similarity of the two expressions may have discouraged students from correctly applying these two expressions to different physical cases.

Step E3: $N=261$ solutions set up the correct expressions for unknown constants in step C3, and the next step E3 uses mathematical calculations to solve these equations for the constants. For the equations to match the boundary conditions, the manipulations are algebraic. For the expression of unknown constants to match the initial conditions [i.e., $\sum_{n} c_{n} \sin (n \pi x / a)=\Psi(x, t=0)$ ], there are two methods available to solve this equation: Fourier transformation and term matching. The Fourier transformation refers to the execution process: multiply the equation by the orthogonal functions, integrate it in the region between the boundaries, and determine the coefficients using the integral result. The term matching involves the execution process: using a trigonometric relation and thus directly comparing the coefficients for the different eigenfunctions in the expansion.

Furthermore, in our exam questions, the same initial conditions were expressed in two different forms: one is a single expression [e.g., $\Psi(x, t=0)=\sqrt{8 / 5 a}(1+\cos \pi x / a) \times$ $\sin \pi x / a]$, and the other is in a superposition form, e.g.,
TABLE VIII. Difficulties in step E3: executing the procedure of solving the constants of the general solution.

\begin{tabular}{lc}
\hline \hline Difficulty & $N$ \\
\hline Erring in the Fourier transformation & 26 \\
Omitting or adding a constant factor & 14 \\
Failing to finish the calculations & 10 \\
Other errors & 3 \\
\hline \hline
\end{tabular}

$$
\Psi(x, t=0)=\sqrt{\frac{8}{5 a}} \sin \frac{\pi x}{a}+\sqrt{\frac{1}{5 a}} \sin \frac{2 \pi x}{a} .
$$

Fourier transformation can be used to address the two cases. On the other hand, the initial conditions offered in the first case can be divided to those for the second case. Thus, term matching can also be used to manipulate the two situations, and the process is considerably more direct and simple compared to the Fourier transformation. However, a large fraction of these exam solutions $(63 \%, N=239$ of 381 ) applied the Fourier transformation, and a small number of these solutions $(6 \%, N=22$ of 381$)$ exploited the term matching.

For calculations of the values of unknown constants, $N=38$ solutions contained various mathematical mistakes (see Table VIII): erring in the Fourier transformation [e.g., not obtaining the correct answer of the integral $\int_{0}^{a} \sin (m \pi x / a) \sin (n \pi x / a) d x$ or the integral $\left.\int_{0}^{a} \sin (n \pi x / a) \Psi(x, 0) d x\right]$, omitting or adding a constant factor (e.g., factor of 2 or length $a$ ), failing to finish the calculations, or other errors (e.g., using $n$ instead of $n^{2}$ for energy eigenvalues).

In the interviews, seventeen participants utilized the Fourier method and only one student used the term matching. Even though the initial condition was given as a sum of $\sin \pi x / a$ and $\sin 2 \pi x / a$, students also exhibited a strong preference for using Fourier transformation instead of simpler term matching. When they were asked why they used the Fourier method, one student explicitly answered, "In our quantum mechanics course we faced various eigenfunctions. We usually expressed them in an integral form to describe their properties, such as the orthogonality $\left[\int_{0}^{a} \varphi_{m}^{*}(x) \varphi_{n}(x) d x=\delta_{m n}\right]$, and used an integral expression to solve the coefficients $\left[c_{n}=\int_{0}^{a} \varphi_{n}^{*}(x) \Psi(x) d x\right]$. This is exactly the Fourier integral method." This typical result suggests that overemphasis on the integral representation of the eigenfunctions may have prevented the students from mastering the eigenfunctions in a different representation and disencouraged them to use term matching as an alternative method.

In addition, there are various issues in the solutions utilizing the Fourier method. This result is expected since the Fourier transformation is a more mathematically demanding strategy, while the term matching only demands a simpler algebraic operation. We note that a similar result 
has been observed in the investigation of students' work on a Laplace equation in the context of electrostatics [33]. Overall, analysis of both the interviews and exam solutions suggests that the mathematical manipulations in step E3 represented the primary barrier to student performance in time evolution problems.

Step E4: The final step E4 in the execution stage compiles the separated spatial and temporal functions of the solution into a single expression. $N=223$ solutions completed step E3, and $N=215$ solutions provided the correct final expression for the wave function. Various mistakes in the remaining $N=8$ solutions included dropping or gaining constant factors, omitting the number $n$ in the energy value term or energy eigenfunction part, or not giving a final expression.

In the interviews, nearly all students who progressed to this step completed the final expression correctly. One student failed to include the $n$ dependence in the expression for the wave function. When this student was asked to justify this expression, he soon modified it and commented, "I spent a lot of time solving this problem. I was a little tired, so I accidentally omitted the subscript $n$. ." This result reflects that the students were exhausted by the lengthy calculations, which may be why they made small errors, such as omitting some terms in the execution.

\section{Reflection on the solutions}

As shown in Sec. III, an expert can use three reflective checks (elements R1-R3) to confirm their solution to time evolution problems. Students' difficulties in the refection stage were relatively difficult to probe and study since many students did not explicitly write down these reflective checks on their solutions. Alternatively, we followed Ref. [33] to review the students' solutions that included errors in the final expressions that could be detected by these checks. However, none of these expressions were modified in the students' solutions. These results suggest that our students rarely used these effective check methods in practice.

In the interviews, all students attempted to check their solutions, but none of them used the three reflective methods. They usually checked their solutions from the first expression to the last expression. In particular, one student suggested, "I directly wrote down a general expression for time evolution of the wave function as $\Psi(x, t)=$ $\sum_{n} c_{n} e^{-i E_{n} t / \hbar} \varphi_{n}(x)$ and compared this form with my solution. I convinced myself that the solution was correct." These results suggest that the students either checked each step of their solutions or produced an expected expression from memory to check the solutions. This situation may account for why effective checks were rare in the students' work.

\section{E. Overview of students' performance}

$N_{t}=381$ students took the exams and were asked to solve the time evolution problem. In Fig. 4 we illustrate the

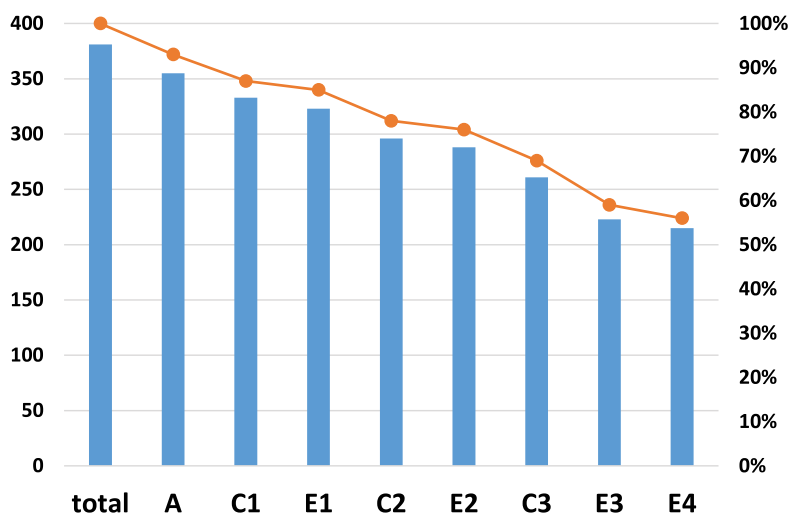

FIG. 4. The correctness of each step as students progressed through the time evolution problem. In the diagram, the histogram denotes the number of correct solutions, and the dots label the correct percentage with respect to the total solutions.

correctness of each step as students progressed through this problem. The histogram denotes the number of correct solutions at each step. The dot is the corresponding correct percentage, which is the fraction of the number of correct solutions $N$ with respect to the number of total solutions $N_{t}=381$. In the end, about $57 \%(N=215$ of 381) successfully passed 8 steps of the problem and provided the correct final answers. As shown in the figure, there has a constant decay of the number of correct solutions, which suggests students struggled with various difficulties in each step. The decay is somewhat larger in step C2, C3, and E3, which indicates there were more issues in these three steps. This result suggests that, in addition to the construction stage, the procedural mathematics requiring the Fourier method can also be a main stumbling block for our students.

\section{CONCLUSIONS AND DISCUSSIONS}

When students used the separation of variables method to solve PDEs in the context of quantum mechanics, they made many, various errors. We focused on two typical types of PDE problems: an energy eigenfunction problem and a time evolution problem. We investigated students' difficulties by examining their solutions to exam questions and conducting "think-aloud" interviews. Then, we identified four broad categories of conceptual and reasoning difficulties within the problem-solving procedure. We followed the ACER framework to analyze the data and organize students' difficulties in the process of applying the separation of variables technique.

\section{A. Findings on energy eigenfunction problems}

Here we summarize our findings with student difficulties organized by the ACER framework. Table IX lists several primary difficulties and possible causes evident in students' responses to the energy eigenfunction problem. Here 
TABLE IX. Primary difficulties of students on solving eigenfunction problem, and the possible causes to which they relate.

\begin{tabular}{|c|c|c|}
\hline Stages & Primary difficulties & Possible causes \\
\hline Activation & Inappropriately using the abstract operator method & $\begin{array}{l}\text { Overfocusing on the abstract formalism which } \\
\text { discourages to activate the PDE tools related to } \\
\text { differential expressions in position representations }\end{array}$ \\
\hline \multirow[t]{4}{*}{ Construction } & $\begin{array}{l}\text { Not setting up the time-independent Schrödinger } \\
\text { equation directly for the eigenfunction problem }\end{array}$ & $\begin{array}{l}\text { Overemphasizing on the time-dependent Schrödinger } \\
\text { equation }\end{array}$ \\
\hline & Selecting two separation constants with incorrect signs & (1) Overgeneralizing the concept of bound states \\
\hline & & $\begin{array}{l}\text { (2) Directly transferring the results of the Laplace } \\
\text { equation to the Schrödinger equation where they are } \\
\text { not applicable }\end{array}$ \\
\hline & $\begin{array}{l}\text { Inappropriately setting up a superposition state } \\
\text { expression }\end{array}$ & $\begin{array}{l}\text { Remembering an algorithm of separation of variables } \\
\text { method but not understanding when and why to } \\
\text { introduce a sum expression }\end{array}$ \\
\hline Execution & Incorrectly separating the PDE into ODEs & $\begin{array}{l}\text { Remembering the separated form of the wave function } \\
\text { rather than understanding the reason for making the } \\
\text { separation constant assumption and justified each step }\end{array}$ \\
\hline Reflection & Rarely using the effective check methods & Developing the habit to check the solutions step by step \\
\hline
\end{tabular}

primary difficulties refer to the errors made by multiple students (typically 20-30 students) in each stage.

\section{B. Findings on time evolution problems}

Table $\mathrm{X}$ lists several primary difficulties and possible explanations evident in students' comments to the time evolution problem.

In summary, we found that students struggled most with the construction stage of the ACER framework. In the construction stage, the students faced two main difficulties: determining the signs of the separation constants, and determining the need of summation form of the wave function. The interview results indicate that the students had not developed a functional understanding of relevant concepts (e.g., energy eigenvalues, superposition states expression) in solving these quantum mechanics problems.

In the execution stage, we found that the process of manipulating the Fourier method was a significant barrier for the students. The interview results suggest that the students had not developed a more fundamental understanding the orthogonal properties of eigenfunctions in quantum mechanics.

\section{Comparison with previous studies on PDE problems in electrostatics}

The previous section presented our findings on students' difficulties with PDEs in the context of quantum mechanics. It is important to compare students' difficulties across

TABLE X. Primary difficulties of students on solving the time evolution problem, and the possible causes to which they relate.

\begin{tabular}{|c|c|c|}
\hline Stages & Primary difficulties & Possible causes \\
\hline Activation & Directly using the final expression & $\begin{array}{l}\text { Remembering the general expression of the time } \\
\text { evolution wave function while not knowing the } \\
\text { explicit procedure to obtain this expression }\end{array}$ \\
\hline \multirow[t]{3}{*}{ Construction } & Selecting the separation constant with incorrect sign & $\begin{array}{l}\text { Not recognizing that the separation constant is } \\
\text { energy constant which should satisfy the criteria } \\
\text { of bound states }\end{array}$ \\
\hline & $\begin{array}{l}\text { Problems in expressing the superposition state to match } \\
\text { the initial condition }\end{array}$ & $\begin{array}{l}\text { Not understanding the fact that a single separated solution } \\
\text { does not satisfy the initial condition while a } \\
\text { superposition form does satisfy }\end{array}$ \\
\hline & $\begin{array}{l}\text { Incorrect belief that the time evolution of a wave function } \\
\text { is always via an overall phase factor } e^{-i E t / \hbar}\end{array}$ & $\begin{array}{l}\text { Confusing the expression } e^{-i E t / \hbar} \varphi(x) \text { for a single } \\
\text { stationary state and the expression } e^{-i E_{n} t / \hbar} \varphi_{n}(x) \\
\text { for a superposition state }\end{array}$ \\
\hline Execution & $\begin{array}{l}\text { Preferring to use Fourier method than term matching and } \\
\text { then making mistakes in Fourier method }\end{array}$ & $\begin{array}{l}\text { Usually use the Fourier integral to express the } \\
\text { orthogonality of eigenfunctions thus not recognizing } \\
\text { alternative methods }\end{array}$ \\
\hline Reflection & Rarely using the effective check methods & $\begin{array}{l}\text { Checking the final answer with the general expression } \\
\text { from memory }\end{array}$ \\
\hline
\end{tabular}


TABLE XI. Primary difficulties of students on solving the Laplace equation in Cartesian coordinates, and the possible causes to which they relate.

\begin{tabular}{|c|c|c|}
\hline Stages & Primary difficulties & Possible causes \\
\hline Activation & (high success) & (none) \\
\hline \multirow[t]{2}{*}{ Construction } & $\begin{array}{l}\text { Selecting incorrect signs for the separation } \\
\text { constants }\end{array}$ & $\begin{array}{l}\text { Overfocusing on satisfying the boundary conditions but not } \\
\text { recognizing that the solution must also satisfy the Laplace } \\
\text { equation }\end{array}$ \\
\hline & $\begin{array}{l}\text { Not setting up a summation form for the } \\
\text { general solution }\end{array}$ & $\begin{array}{l}\text { Using pattern matching to address similar problems without } \\
\text { being able to justify complete steps }\end{array}$ \\
\hline \multirow[t]{3}{*}{ Execution } & $\begin{array}{l}\text { Preferring to use Fourier method than term } \\
\text { matching and then making mistakes in } \\
\text { Fourier method }\end{array}$ & $\begin{array}{l}\text { (i) Strongly linking the canonical kinds of boundary conditions } \\
\text { in the Cartesian coordinates to Fourier method }\end{array}$ \\
\hline & & $\begin{array}{l}\text { (ii) Not internalizing the properties of orthogonal functions } \\
\text { enough to see term matching as a viable strategy }\end{array}$ \\
\hline & & $\begin{array}{l}\text { (Note that since there were no interview results, the above two } \\
\text { possible causes were only speculations by the researchers) }\end{array}$ \\
\hline Reflection & $\begin{array}{l}\text { Rarely spontaneously attempting to reflect } \\
\text { the final answers }\end{array}$ & (No interview results) \\
\hline
\end{tabular}

contexts to see what similarities and differences there are. As mentioned in the section of literature overview, Wilcox et al. studied students' difficulties with solving the Laplace equation in Cartesian coordinates in the context of electrostatics [33]. For comparison, we summarized their research findings in Table XI.

(i) Activation stage: Wilcox et al. found that students were highly successful in recognizing separation of variables as the appropriate mathematical technique to solve the Laplace equation [33].

In contrast, in our study, students were less successful in recognizing separation of variables to solve the Schrödinger equation in high spatial dimensions. This may be, in part, due to the student tendency to active the operator method for quantum mechanics problems.

(ii) Construction stage: Wilcox et al. found a primary issue was that students selected incorrect signs for the separation constants. This error was possibly because they were overfocusing on satisfying the boundary conditions while not considering that the solution must also satisfy the Laplace equation [33]. Another primary issue was that students could not recall or justify the need for the summation form of the general solution. One potential explanation of this issue was that students used pattern matching to approach these problems without being able to justify complete steps [33].

These difficulties also appeared in our study in the context of quantum mechanics. However, the possible explanations are largely different (see the summary text and tables in the previous section). These differences are understandable since the construction stage of problem-solving process is highly dependent on the specific physics context of what the problem describes.

Moreover, in our study, one issue is to distinguish between different application scenarios of timedependent Schrödinger equation and time-independent Schrödinger equation. However, as students were typically not required to distinguish between the Laplace equation and alternative equation or they were explicitly given the Laplace equation, this difficulties was not observed in the studies for the electrostatics problems [33].

In particular, a primary difficulty on timedependent problem is that students incorrectly believed that the time evolution of a wave function is always via an overall phase factor $e^{-i E t / \hbar}$. This difficulty is unique to the context of quantum mechanics and certainly does not appear in other physics contexts.

(iii) Execution stage: Wilcox et al. found student tendency to use Fourier method over term matching for solving unknown constants when both strategies were possible. Without interview data on student reasoning, the researchers suspected that this may be due to the canonical kinds of boundary conditions in the Cartesian geometries, or because students did not understand the properties of orthogonal functions enough to see other method as a viable strategy [33].

This tendency was also observed in our study. In fact, we explicitly probed student reasoning on this issue in the interviews. The students' responses in the interviews support the possible explanation that they preferred to use the Fourier integral to express the orthogonality of eigenfunctions in the context of quantum mechanics. Thus students did not connect 
the Fourier method and term matching as related strategies and preferred one method over the other.

(iv) Reflection stage: Wilcox et al. found that very few of students made spontaneous attempts to reflect on their final answers [33]. They did not give explanations for this issue.

In contrast to their results, in our study, we found that students usually reflected on their solutions step by step despite never using effective check methods. Students explicitly commented that this habit was developed through various examinations over the years.

In summary, in the construction stage to solve a PDE problem in physics courses, the students faced several difficulties which are content specific. It is understandable since in this stage the students were required to convert a physics situation into a mathematical representation (e.g., set up a PDE or match the boundary conditions) or explain the physics meaning of the results of a mathematical procedure (e.g., physical significance of separation constants). While in the execution stage, the students encountered a main difficulty to understand the properties of orthogonal functions and master the Fourier method and related method, which is largely content independent.

\section{Discussion on student learning of similar topics in different contexts}

It is usually assumed that when students are exposed to certain techniques of solving problems in one context, they will be able to transfer their knowledge and skills from one physics context to another. However, this did not happen in our study. Our findings agree with previous studies which suggested that the positive transfer of problem-solving skills across different contexts is rare [36,37,53]. In a traditional curriculum at USTC, students were often first exposed to the application of PDE tools in their mathematics course and electromagnetism course, before they took quantum mechanics. The separation of variables method and related PDE tools were discussed several times in these courses and thus much time was devoted to this topic. To be precise, there are two weeks spent on the Laplace equation (4 sessions/week, $50 \mathrm{~min} /$ session), followed by 5 or 6 homework problems. Despite many opportunities to practice the PDE tools in the electromagnetism context, students did not get enough mastery of these techniques in the new context of quantum mechanics. As compared to the previous study in the context of electrostatics [33], our study demonstrated that students' difficulties can perpetuate and new difficulties can occur when students transfer certain techniques of solving PDE problems from one context to another.

In addition, previous studies indicated that students might experience "interference" of their knowledge and understanding between different contexts [36,54]. The interference refers to the cognitive process that the past learned memories and thoughts would have a negative influence in comprehending a similar topic in new context. In the present study, we observed one example of such interference. As mentioned in step $\mathrm{C} 2$ of Sec. V, one interview student tried to decide the signs of two separation constants for a Schrödinger equation and selected one is a positive value and the other is a negative value. When asked by the researcher why he made such choice, the student explicitly explained he remembered the choice from his experience about the Laplace equation in electrostatics. Because they usually learn the electromagnetism course first, students have much more experience with PDE tools in the context of electrostatics, long before they see it as part of quantum mechanics. As a consequence, one can expect the interference in applying PDE tools in quantum mechanics context from electrostatics context.

\section{E. Comparison with studies on time evolution problems}

Previous studies have investigated students' understanding of the time dependence of a wave function. In Ref. [10], students from seven universities were given a linear superposition of the ground and first excited state wave function as the initial wave function [e.g., $\left.\Psi(x, 0)=\sqrt{\frac{2}{7}} \varphi_{1}(x)+\sqrt{\frac{5}{7}} \varphi_{2}(x)\right]$ and asked to find the wave function after a time $t$. The researchers found that many students wrote a common phase factor for both terms [i.e., $\left.\Psi(x, t)=e^{-i E t / \hbar} \Psi(x, 0)\right]$ instead of the correct answer.

In another study [13], students at the University of Washington were given a task to find the wave function at time $t$ for two possible initial states: $\Psi(x, 0)=\varphi_{2}(x)$, or $\Psi(x, 0)=\sqrt{\frac{1}{2}}\left[\varphi_{1}(x)+\varphi_{2}(x)\right]$. The researchers found the most prominent error on student understanding of time evolution is a tendency to associate a single time dependent phase with the entire wave function, rather than to associate an individual phase with each term. For example, some students wrote a wave function as $\Psi(x, t)=\sqrt{\frac{1}{2}} e^{-i E t / \hbar}\left[\varphi_{1}(x)+\varphi_{2}(x)\right]$.

Interestingly, we found that students' understanding of stationary state could also affect their performance in solving the time-dependent Schrödinger equation. From both the exam solutions and student interviews on problem 2 , we observed that some students wrote down the time evolution of a wave function via an overall phase factor $e^{-i E t / \hbar}$ (for details, please see the examples in step C 3 and E2 in Sec. V). One hypothesis is students confused the expression $e^{-i E t / \hbar} \varphi(x)$ for a single energy eigenfunction and the expression $e^{-i E_{n} t / \hbar} \varphi_{n}(x)$ for each component in a superposition state of energy eigenfunctions. Therefore, the students' excessive focus on the stationary state could be hindering them from applying the superposition state expression correctly.

In summary, the tendency to treat the time dependence of all wave functions as having a single phase seems to be 
persistent in student reasoning for solving both the nonalgorithmic problems (as documented in prior studies $[10,13]$ ) and algorithmic problems (as documented in the present paper).

\section{F. Relation with studies on activating resources}

In Ref. [55], Frank et al. investigated how students thought about simple harmonic and projectile motion questions in the context of classical mechanics. Their data show that different cues included in two questions about a single physical situation resulted in variations in the distribution of student answers. For example, the distance-cuing questions were more likely to elicit an answer based on a distance-time resource. While the speed-cuing questions about the same physical situation would activate the speed-related resource. Therefore, the researchers assumed that the specifics of how questions are posed influences which of the many resources are activated.

Interestingly, we also observed an example of variation in the pattern of students' responses when details of the problems asked are changed. In some interviews, the same type of eigenfunction problem as shown in Fig. 1 was given to students with minor differently constructed prompts [e.g., explicitly wording "boundary condition $\Psi(x=a, y)$ "]. In these cases, the interviewed students all used the separation of variables method. One student explained, "the separation of variables method is usually applied to solve the PDE problems with explicitly given boundary conditions." Thus, it is possible that when being prompted explicitly to use the boundary conditions, students usually activated the resources of separation of variables.

\section{G. Implications for instruction}

Our investigation of students' common difficulties can provide several implications for instruction on regarding PDEs in quantum mechanics.

First, for the time evolution problem, the students had difficulty spontaneously using the separation of variables method. It is important to target this issue for students to a more robust understanding of the general expression of the time evolution wave function instead of directly memorizing it for exams. The students also had difficulty in understanding the reason to use a linear combination form of the separated solutions to construct the general solution. It is important to focus on this critical issue in order for students to form a clearer conception of the general expression of the time evolution wave function. For example, it is particularly effective to ask the students to try which of the two approaches can match the initial condition: using the form of a single stationary state $e^{-i E t / \hbar} \varphi(x)$ or using the form of a linear combination of energy eigenfunctions $\sum_{n} c_{n} e^{-i E_{n} t / \hbar} \varphi_{n}(x)$.
As noted in Ref. [33], the students commonly solved PDEs through pattern matching with previous similar problems. We found that several variations of these questions can encourage students to grasp general method of separation of variables, rather than simply using pattern matching from memory. For example, providing a twodimensional potential instead of a simple one-dimensional potential can force students to attempt the separation of variables procedure. It is also helpful to provide an opportunity for students to compare when the Fourier method or term matching is more efficient for solving the unknown constants.

In this work, we utilized the ACER framework to organize and analyze the student problem-solving process when dealing with two typical PDEs in quantum mechanics. This framework can be used to probe and study students' works in other contexts in quantum mechanics courses. Additional studies can provide a wide perspective on the student problem-solving process, allowing instructors to design different instructional strategies and curricular materials to address student understanding and reasoning difficulties in this process.

\section{ACKNOWLEDGMENTS}

We thank Professor A. M. Chang at Duke University and Professor H. W. Jiang at UCLA for their helpful discussion and assistance. This work was supported by the Education Research Foundation of Anhui Provincial and the National Natural Science Foundation of China (No. 11974336).

\section{APPENDIX A: OPERATIONALIZATION OF THE ACER FRAMEWORK FOR QUESTION 1}

In the following we provide the summaries of the process used to solve problem 1 (the energy eigenvalue problem in Fig. 1) according to the ACER framework.

- Step A.-The basic equation is a PDE in the twodimensional space region, which can be solved by the separation of variables method.

- Step C1.-Express the basic equation for energy eigenfunctions:

Inside the box, the time-independent Schrödinger equation reads

$$
-\frac{\hbar^{2}}{2 m}\left(\frac{\partial^{2}}{\partial x^{2}}+\frac{\partial^{2}}{\partial y^{2}}\right) \Psi(x, y)=E \Psi(x, y) .
$$

- Step E1.-Using a separated form of wave function to divide the PDE into two ODEs:

We look for the solution as $\Psi(x, y)=f(x) g(y)$ and plug it into the PDE. Using differential calculations and introducing the separation constants $E_{1}$ and $E_{2}$, we obtain 
$-\frac{\hbar^{2}}{2 m} \frac{d^{2} f(x)}{d x^{2}}=E_{1} f(x), \quad-\frac{\hbar^{2}}{2 m} \frac{d^{2} g(y)}{d y^{2}}=E_{2} g(y)$,

with $E_{1}+E_{2}=E$.

- Step C2.-Choose the signs of the separation constants:

We assume $E_{1}=\hbar^{2} k_{1}^{2} / 2 m>0$ and $E_{2}=\hbar^{2} k_{1}^{2} /$ $2 m>0$; then, the ODEs are expressed as

$$
\frac{d^{2} f}{d x^{2}}=-k_{1}^{2} f, \quad \frac{d^{2} g}{d y^{2}}=-k_{2}^{2} g .
$$

- Step E2.-Provide the solutions to the ODEs:

The above equations have the following solutions

$$
\begin{aligned}
& f(x)=A \sin k_{1} x+B \cos k_{1} x, \\
& g(y)=C \sin k_{2} y+D \cos k_{2} y,
\end{aligned}
$$

where $A, B, C$, and $D$ are the unknown constants.

- Step C3(i).-Setup the equations for all unknown constants:

A general solution for the PDE is $\Psi(x, y)=f(x) g(y)=\left(A \sin k_{1} x+B \cos k_{1} x\right)\left(C \sin k_{2} y+\right.$ $\left.D \cos k_{2} y\right)$. Applying the boundary conditions, we have

$$
\begin{gathered}
f(x=0)=f(x=a)=0, \\
g(y=0)=g(y=b)=0 .
\end{gathered}
$$

- Step E3(i).-Determine the energy eigenvalues:

Using algebraic calculations, we obtain $k_{1}=n_{1} \pi / a$ and $k_{2}=n_{2} \pi / b$, where $n_{1}$ and $n_{2}$ are integers. Hence,

$$
f(x)=A \sin k_{1} x, \quad g(y)=C \sin k_{2} y .
$$

- Step C3(ii).-Setup the equations for all unknown constants:

Applying the normalization of the wave function, we have

$$
\int_{0}^{a}\left|A \sin k_{1} x\right|^{2} d x=1, \quad \int_{0}^{b}\left|C \sin k_{2} x\right|^{2} d x=1 .
$$

- Step E3(ii).-Determine other constants:

Using integral calculations, we have

$$
A=\sqrt{2 / a}, \quad C=\sqrt{2 / b} .
$$

- Step E4.-Express the final answer:

Inside the box, the energy eigenfunctions are

$$
\Psi_{n_{1} n_{2}}(x, y)=\sqrt{\frac{4}{a b}} \sin \left(\frac{n_{1} \pi x}{a}\right) \sin \left(\frac{n_{2} \pi y}{b}\right) .
$$

- Step R.-Use the effective reflection methods to check the final answer.

\section{APPENDIX B: OPERATIONALIZATION OF THE ACER FRAMEWORK FOR QUESTION 2}

Following the ACER framework, summaries of the process used to calculate the wave function for problem 2 (the time evolution problem in Fig. 2) are shown here.

- Step A.-The basic equation is a PDE in the twodimensional space-time region, which prompts the application of the separation of variables method.

- Step C1.-Express the basic equation for the time evolution process:

For a particle in a one-dimensional infinite square well, the time-dependent Schrödinger equation reads

$$
i \hbar \frac{\partial}{\partial t} \Psi(x, t)=-\frac{\hbar^{2}}{2 m} \frac{\partial^{2}}{\partial x^{2}} \Psi(x, t) .
$$

- Step E1.-Use a separated form of wave function to divide the PDE into two ODEs:

We look for the solution to $\Psi(x, t)=\varphi(x) f(t)$ and insert it into the PDE. With differential calculations and introducing the separation constant $E$, we obtain

$$
i \hbar \frac{d f(t)}{d t}=E f(t), \quad-\frac{\hbar^{2}}{2 m} \frac{d^{2} \varphi(x)}{d x^{2}}=E \varphi(x) .
$$

- Step C2.-Choose the sign of the separation constant: We assume $E=\hbar^{2} k^{2} / 2 m>0$; then, the ODEs are expressed as

$$
\frac{d f}{d t}=\frac{-i E}{\hbar} f, \quad \frac{d^{2} \varphi}{d x^{2}}=-k^{2} \varphi .
$$

- Step E2.-Provide the solutions to the ODEs:

It is easy to solve the first ODE and obtain the solution as

$$
f(t)=e^{-i E t / \hbar} .
$$

The second ODE has the following solution

$$
\varphi(x)=A \sin k_{1} x+B \cos k_{1} x,
$$

where $A$ and $B$ are the unknown constants.

- Step C3(i).--Setup the equations for all unknown constants:

Applying the boundary conditions, we have

$$
\varphi(x=0)=\varphi(x=a)=0 .
$$


- Step E3(i).-Determine the energy eigenvalues:

Using algebraic calculations, we find $k=n \pi / a$ with integers $n$. Hence,

$$
\varphi(x)=A \sin k x .
$$

- Step C3(ii).-Setup the equations for all unknown constants:

Applying the normalization of the wave function, we have

$$
\int_{0}^{a}|A \sin k x|^{2} d x=1 .
$$

- Step E3(ii).-Determine other constants:

Using integral calculations, we obtain

$$
A=\sqrt{2 / a} .
$$

- Step C3(iii).--Set up the equations for all unknown constants:

A general solution for the PDE is a linear combination of the separable solutions

$\Psi(x, t)=\sum_{n} c_{n} f(t) \varphi(x)=\sum_{n} c_{n} e^{-i E_{n} t / \hbar} \sqrt{\frac{2}{a}} \sin \left(\frac{n \pi x}{a}\right)$.

[1] C. Singh, Student understanding of quantum mechanics, Am. J. Phys. 69, 885 (2001).

[2] C. Singh, M. Belloni, and W. Christian, Improving students' understanding of quantum mechanics, Phys. Today 59, No. 8, 43 (2006).

[3] C. Singh, Student understanding of quantum mechanics at the beginning of graduate instruction, Am. J. Phys. 76, 277 (2008).

[4] S. B. McKagan, K. K. Perkins, and C. E. Wieman, Deeper look at student learning of quantum mechanics: The case of tunneling, Phys. Rev. ST Phys. Educ. Res. 4, 020103 (2008).

[5] G. Zhu and C. Singh, Improving students' understanding of quantum mechanics via Stern-Gerlach experiment, Am. J. Phys. 79, 499 (2011).

[6] G. Zhu and C. Singh, Surveying students' understanding of quantum mechanics in one spatial dimension, Am. J. Phys. 80, 252 (2012).

[7] G. Zhu and C. Singh, Improving students' understanding of quantum measurement I: Investigation of difficulties, Phys. Rev. ST Phys. Educ. Res., 8, 010117 (2012).

[8] G. Zhu and C. Singh, Improving students' understanding of quantum measurement II: Development of Researchbased learning tools, Phys. Rev. ST Phys. Educ. Res. 8, 010118 (2012).

[9] G. Zhu and C. Singh, Improving student understanding of addition of angular momentum in quantum mechanics, Phys. Rev. ST Phys. Educ. Res. 9, 010101 (2013).
Applying the initial condition, we have

$$
\begin{aligned}
\Psi(x, 0) & =\sqrt{\frac{8}{5 a}}\left(1+\cos \frac{\pi x}{a}\right) \sin \frac{\pi x}{a} \\
& =\sum_{n} c_{n} \sqrt{\frac{2}{a}} \sin \left(\frac{n \pi x}{a}\right) .
\end{aligned}
$$

- Step E3(iii).-Determine the coefficients:

Utilizing the Fourier method or term matching, we obtain $c_{1}=\sqrt{\frac{4}{5}}, c_{2}=\sqrt{\frac{1}{5}}$, and $c_{n}=0(n \neq 1,2)$.

- Step E4.-Express the final answer:

Inside the well, the wave function at time $t$ is

$$
\begin{aligned}
\Psi(x, t)= & \sqrt{\frac{4}{5}} e^{-i E_{1} t / \hbar} \sqrt{\frac{2}{a}} \sin \left(\frac{\pi x}{a}\right) \\
& +\sqrt{\frac{1}{5}} e^{-i E_{2} t / \hbar} \sqrt{\frac{2}{a}} \sin \left(\frac{2 \pi x}{a}\right) .
\end{aligned}
$$

- Step R.-Apply the effective reflection methods to check the final answer.
[10] C. Singh and E. Marshman, Review of student difficulties in upper-level quantum mechanics, Phys. Rev. ST Phys. Educ. Res. 11, 020117 (2015).

[11] E. Marshman and C. Singh, Framework for understanding the patterns of student difficulties in quantum mechanics, Phys. Rev. ST Phys. Educ. Res. 11, 020119 (2015).

[12] G. Passante, P. J. Emigh, and P. S. Shaffer, Examining student ideas about energy measurements on quantum states across undergraduate and graduate levels, Phys. Rev. ST Phys. Educ. Res. 11, 020111 (2015).

[13] P. J. Emigh, G. Passante, and P. S. Shaffer, Student understanding of time dependence in quantum mechanics, Phys. Rev. ST Phys. Educ. Res. 11, 020112 (2015).

[14] E. Gire and E. Price, Structural features of algebraic quantum notations, Phys. Rev. ST Phys. Educ. Res. 11, 020109 (2015).

[15] C. Baily and D. N. Finkelstein, Teaching quantum interpretations: Revisiting the goals and practices of introductory quantum physics, Phys. Rev. ST Phys. Educ. Res. 11, 020124 (2015).

[16] K. Krijtenburg-Lewerissa, H. J. Pol, A. Brinkman, and W. R. van Joolingen, Insights into teaching quantum mechanics in secondary and lower undergraduate education, Phys. Rev. Phys. Educ. Res. 13, 010109 (2017).

[17] E. Marshman and C. Singh, Investigating and improving student understanding of quantum mechanics in the context 
of single photon interference, Phys. Rev. Phys. Educ. Res. 13, 010117 (2017).

[18] R. Sayer, A. Maries, and C. Singh, Quantum interactive learning tutorial on the double-slit experiment to improve student understanding of quantum mechanics, Phys. Rev. Phys. Educ. Res. 13, 010123 (2017).

[19] A. Maries, R. Sayer, and C. Singh, Effectiveness of interactive tutorials in promoting "which-path" information reasoning in advanced quantum mechanics, Phys. Rev. Phys. Educ. Res. 13, 020115 (2017).

[20] J. R. Hoehn and N. D. Finkelstein, Students' flexible use of ontologies and the value of tentative reasoning: Examples of conceptual understanding in three canonical topics of quantum mechanics, Phys. Rev. Phys. Educ. Res. 14, 010122 (2018).

[21] P. J. Emigh, G. Passante, and P. S. Shaffer, Developing and assessing tutorials for quantum mechanics: Time dependence and measurements, Phys. Rev. Phys. Educ. Res. 14, 020128 (2018).

[22] T. Wan, P. J. Emigh, and P. S. Shaffer, Investigating how students relate inner products and quantum probabilities, Phys. Rev. Phys. Educ. Res. 15, 010117 (2019).

[23] T. Wan, P. J. Emigh, and P. S. Shaffer, Probing student reasoning in relating relative phase and quantum phenomena, Phys. Rev. Phys. Educ. Res. 15, 020139 (2019).

[24] C. D. Porter and A. F. Heckler, Graduate student misunderstandings of wave functions in an asymmetric well, Phys. Rev. Phys. Educ. Res. 15, 010139 (2019).

[25] B. P. Schermerhorn, G. Passante, H. Sadaghiani, and S. J. Pollock, Exploring student preferences when calculating expectation values using a computational features framework, Phys. Rev. Phys. Educ. Res. 15, 020144 (2019).

[26] G. Passante and A. Kohnle, Enhancing student visual understanding of the time evolution of quantum systems, Phys. Rev. Phys. Educ. Res. 15, 010110 (2019).

[27] B. Modir, J. D. Thompson, and E. C. Sayre, Framing difficulties in quantum mechanics, Phys. Rev. Phys. Educ. Res. 15, 020146 (2019).

[28] S. Y. Lin and C. Singh, Categorization of quantum mechanics problems by professors and students, Eur. J. Phys. 31, 57 (2010).

[29] B. Modir, J. D. Thompson, and E. C. Sayre, Students' epistemological framing in quantum mechanics problem solving, Phys. Rev. Phys. Educ. Res. 13, 020108 (2017).

[30] C. L. Rasmussen and K. D. King, Locating starting points in differential equations: A realistic mathematics education approach, Int. J. Math. Educ. Sci. Technol. 31, 161 (2000).

[31] S. Habre, Exploring students' strategies to solve ordinary differential equations in a reformed setting, J. Math. Behav. 18, 455 (2000).

[32] C. L. Rasmussen, New directions in differential equations: A framework for interpreting students' understandings and difficulties, J. Math. Behav. 20, 55 (2001).

[33] B. R. Wilcox and S. J. Pollock, Upper-division student difficulties with separation of variables., Phys. Rev. ST Phys. Educ. Res. 11, 020131 (2015).

[34] B. R. Wilcox, M.D. Caballero, D. A. Rehn, and S. J. Pollock, Analytic framework for students' use of mathematics in upper-division physics, Phys. Rev. ST Phys. Educ. Res. 9, 020119 (2013).
[35] B. R. Wilcox and S. J. Pollock, Upper-division student difficulties with the Dirac delta function, Phys. Rev. ST Phys. Educ. Res. 11, 010108 (2015).

[36] Q.X. Ryan, B. R. Wilcox, and S. J. Pollock, Student difficulties with boundary conditions in the context of electromagnetic waves, Phys. Rev. ST Phys. Educ. Res. 14, 020126 (2018).

[37] J. P. Zwolak and C. A. Manogue, Assessing student reasoning in upper-division electricity and magnetism at oregon state university, Phys. Rev. ST Phys. Educ. Res. 11, 020125 (2015).

[38] M. E. Loverude and B.S. Ambrose, Editorial: Focused collection: PER in upper-division physics courses., Phys. Rev. ST Phys. Educ. Res. 11, 020002 (2015).

[39] E. C. Sayre and M. C. Wittmann, Plasticity of intermediate mechanics students' coordinate system choice, Phys. Rev. ST Phys. Educ. Res. 4, 020105 (2008).

[40] C. S. Wallace and S. V. Chasteen, Upper-division students' difficulties with Ampere's law, Phys. Rev. ST Phys. Educ. Res. 6, 020115 (2010).

[41] D.-H. Nguyen and N. S. Rebello, Students' difficulties with integration in electricity, Phys. Rev. ST Phys. Educ. Res. 7, 010113 (2011).

[42] R. E. Pepper, S. V. Chasteen, S. J. Pollock, and K. K. Perkins, Observations on student difficulties with mathematics in upper-division electricity and magnetism, Phys. Rev. ST Phys. Educ. Res. 8, 010111 (2012).

[43] E. B. Pollock, J. R. Thompson, and D. B. Mountcastle, Student understanding of the physics and mathematics of process variables in $\mathrm{P}-\mathrm{V}$ diagrams, AIP Conf. Proc. 951, 168 (2007).

[44] M. E. Loverude, Student understanding of basic probability concepts in an upper-division thermal physics course, AIP Conf. Proc. 1179, 189 (2009).

[45] M. E. Loverude, Investigating student understanding for a statistical analysis of two thermally interacting solids, AIP Conf. Proc. 1289, 213 (2010).

[46] T. I. Smith, J. R. Thompson, and D. B. Mountcastle, Student understanding of Taylor series expansions in statistical mechanics, Phys. Rev. ST Phys. Educ. Res. 9, 020110 (2013).

[47] T. J. Bing and E. F. Redish, Analyzing problem solving using math in physics: Epistemological framing via warrants, Phys. Rev. ST Phys. Educ. Res. 5, 020108 (2009).

[48] R. R. Bajracharya, P. J. Emigh, and C. A. Manogue, Students' strategies for solving a multirepresentational partial derivative problem in thermodynamics, Phys. Rev. Phys. Educ. Res. 15, 020124 (2019).

[49] M. D. Caballero, B. R. Wilcox, L. Doughty, and S. J. Pollock, Unpacking students' use of mathematics in upper-division physics, Eur. J. Phys. 36, 065004 (2015).

[50] J. Y. Zeng, Quantum Mechanics (Science Press, Beijing, 2008).

[51] D. J. Griffiths, Introduction to Quantum Mechanics (Pearson Prentice Hall, New Jersey, 2004).

[52] M. T. H. Chi, in The Thinking Aloud Method, edited by M. W. van Someren, Y. F. Barnard, and J. A. C. Sandberg (Academic Press, London, 1994), Chap. 1. 
[53] S. K. Reed, G. W. Ernst, and R. Banerji, The role of analogy in transfer between similar problem states, Cogn. Psychol. 6, 436 (1974).

[54] T. D. Tomlinson, D. E. Huber, C. A. Rieth, and E. J. Davelaar, An interference account of cue-independent forgetting in the no-think paradigm, Proc. Natl. Acad. Sci. U.S.A. 106, 15588 (2009).

[55] B. W. Frank, S. E. Kanim, and L. S. Gomez, Accounting for variability in student responses to motion questions, Phys. Rev. ST Phys. Educ. Res. 4, 020102 (2008). 